AIP Appilied Physics

\title{
Nanoscale density fluctuations in swift heavy ion irradiated amorphous $\mathrm{SiO} 2$
}

P. Kluth, O. H. Pakarinen, F. Djurabekova, R. Giulian, M. C. Ridgway et al.

Citation: J. Appl. Phys. 110, 123520 (2011); doi: 10.1063/1.3671614

View online: http://dx.doi.org/10.1063/1.3671614

View Table of Contents: http://jap.aip.org/resource/1/JAPIAU/v110/i12

Published by the American Institute of Physics.

\section{Related Articles}

Helium irradiation effects in polycrystalline $\mathrm{Si}$, silica, and single crystal $\mathrm{Si}$

J. Appl. Phys. 111, 083527 (2012)

Tunable nanometer electrode gaps by $\mathrm{MeV}$ ion irradiation

Appl. Phys. Lett. 100, 153108 (2012)

Freestanding nanostructures for three-dimensional superconducting nanodevices

Appl. Phys. Lett. 100, 143106 (2012)

Atomic-scale effects behind structural instabilities in Si lamellae during ion beam thinning AIP Advances 2, 012186 (2012)

Controlling dopant profiles in hyperdoped silicon by modifying dopant evaporation rates during pulsed laser melting

Appl. Phys. Lett. 100, 112112 (2012)

\section{Additional information on J. Appl. Phys.}

Journal Homepage: http://jap.aip.org/

Journal Information: http://jap.aip.org/about/about_the_journal

Top downloads: http://jap.aip.org/features/most_downloaded

Information for Authors: http://jap.aip.org/authors

\section{ADVERTISEMENT}

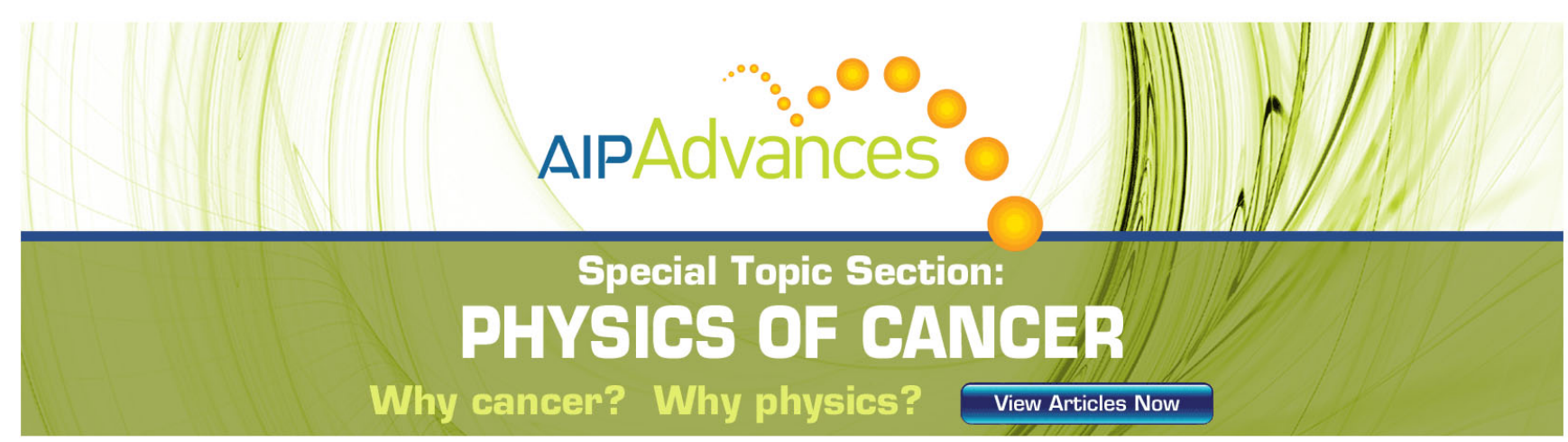




\title{
Nanoscale density fluctuations in swift heavy ion irradiated amorphous $\mathrm{SiO}_{2}$
}

\author{
P. Kluth, ${ }^{1, a)}$ O. H. Pakarinen, ${ }^{2}$ F. Djurabekova, ${ }^{2}$ R. Giulian, ${ }^{1}$ M. C. Ridgway, ${ }^{1}$ A. P. Byrne,${ }^{3}$ \\ and K. Nordlund ${ }^{2}$ \\ ${ }^{1}$ Department of Electronic Materials Engineering, Australian National University, Canberra ACT 0200, \\ Australia \\ ${ }^{2}$ Department of Physics and Helsinki Institute of Physics, University of Helsinki, Helsinki, Finland \\ ${ }^{3}$ Department of Nuclear Physics, Australian National University, Canberra ACT 0200, Australia
}

(Received 20 July 2011; accepted 18 November 2011; published online 23 December 2011)

\begin{abstract}
We report on the observation of nanoscale density fluctuations in $2 \mu$ m thick amorphous $\mathrm{SiO}_{2}$ layers irradiated with $185 \mathrm{MeV} \mathrm{Au}$ ions. At high fluences, in excess of approximately $5 \times 10^{12}$ ions $/ \mathrm{cm}^{2}$, where the surface is completely covered by ion tracks, synchrotron small angle $\mathrm{x}$-ray scattering measurements reveal the existence of a steady state of density fluctuations. In agreement with molecular dynamics simulations, this steady state is consistent with an ion track "annihilation" process, where high-density regions generated in the periphery of new tracks fill in low-density regions located at the center of existing tracks. (C) 2011 American Institute of Physics. [doi:10.1063/1.3671614]
\end{abstract}

\section{INTRODUCTION}

The interaction of swift heavy ions (SHIs) with matter is governed by their inelastic collisions with target electrons. The resulting intense electronic excitation and the concomitant high energy deposition can lead to intriguing material modifications mediated by the formation of narrow defect regions along the ion paths, so called "ion tracks." Ion tracks are of fundamental interest in a wide variety of scientific areas including materials science and engineering, nuclear physics, geochronology, archaeology, and interplanetary science. ${ }^{1}$ Examples of material modifications by SHI irradiation include stabilization of a high pressure phase in $\mathrm{Gd}_{2} \mathrm{Zr}_{2} \mathrm{O}_{7}{ }^{2}$ densification and plastic deformation in amorphous materials such as $\mathrm{SiO}_{2}$ and glassy metals, ${ }^{3,4}$ and amorphisation of crystalline materials (for example, InP, ${ }^{5}$ quartz-SiO $2,{ }^{4,6}$ and natural apatite ${ }^{7}$ ). Recently, a process by which spherical metallic nanoparticles, embedded in amorphous $\mathrm{SiO}_{2}\left(\mathrm{a}-\mathrm{SiO}_{2}\right)$, elongate to form nanorods under SHI irradiation has received significant attention. ${ }^{8-14}$ This process was shown to be closely related to ion track formation in $\mathrm{a}-\mathrm{SiO}_{2}$ and requires multiple track overlap. The plastic deformation, also termed "ion hammering," that is facilitated by ion track formation can be observed macroscopically by using microscopy, ${ }^{15}$ however, underlying changes to the micro-structure are difficult to retrieve due to the lack of sufficient contrast inherent with most analytical techniques.

Small angle $\mathrm{x}$-ray scattering (SAXS) provides an efficient and effective technique to study ion tracks. Using synchrotron SAXS measurements, we recently demonstrated that isolated ion tracks in $\mathrm{a}-\mathrm{SiO}_{2}$ exhibit a previously unresolved fine structure in the radial density distribution comprising a cylindrical core-shell configuration with a lower density core and a higher density shell as compared to un-

\footnotetext{
a) Author to whom correspondence should be addressed. Electronic mail: patrick.kluth@anu.edu.au.
}

irradiated material. ${ }^{16,17}$ In this work we show that SAXS measurements and molecular dynamics (MD) simulations are consistent with a steady state of nano-scale density fluctuations that arise when a- $\mathrm{SiO}_{2}$ is irradiated with SHIs at fluences sufficient to cover the entire surface with ion tracks. This steady state can be explained in terms of an ion track annihilation process; while the SHI induced densification of a-SiO ${ }_{2}$ has been widely reported (for example, see Ref. 4), we show that the compacted layer is not homogeneous but instead is characterized by density fluctuations on a nanometer length scale. The latter result from track overlap where newly formed tracks (partially) annihilate pre-existing tracks.

\section{EXPERIMENTAL AND COMPUTATIONAL METHODS}

Samples were produced by irradiating $2 \mu \mathrm{m}$ thick thermally grown a-SiO $\mathrm{Si}_{2}$ on $\mathrm{Si}$ with $185 \mathrm{MeV} \mathrm{Au}$ ions at the ANU Heavy Ion Accelerator Facility. Fluences ranged between $6 \times 10^{10}$ and $3 \times 10^{14}$ ions $/ \mathrm{cm}^{2}$. Irradiation was performed at room temperature with the incident ion direction normal to the sample surface. Thin $\mathrm{SiO}_{2}$ layers were utilized to achieve a reasonably uniform energy loss over the extent of the layer. Synchrotron SAXS measurements in transmission geometry were performed at beamline 15ID-D of the Advanced Photon Source, Argonne National Laboratories, USA, and the SAXS/WAXS beamline at the Australian Synchrotron in Melbourne, Australia, using X-ray energies of 10.27 and $12 \mathrm{keV}$, respectively. Samples were prepared by locally removing the $\mathrm{Si}$ substrate to isolate the thin $\mathrm{SiO}_{2}$ film. ${ }^{17}$ This enables measurements on the thin film only and negates artifacts due to irradiation induced inhomogeneities in the Si substrate. Measurements were performed with the sample surface aligned normal to the x-ray beam, i.e., parallel to the ion tracks. The resulting isotropic images were radially integrated around the beam center. Scattering from an unirradiated $\mathrm{SiO}_{2}$ standard was subtracted from all spectra. 


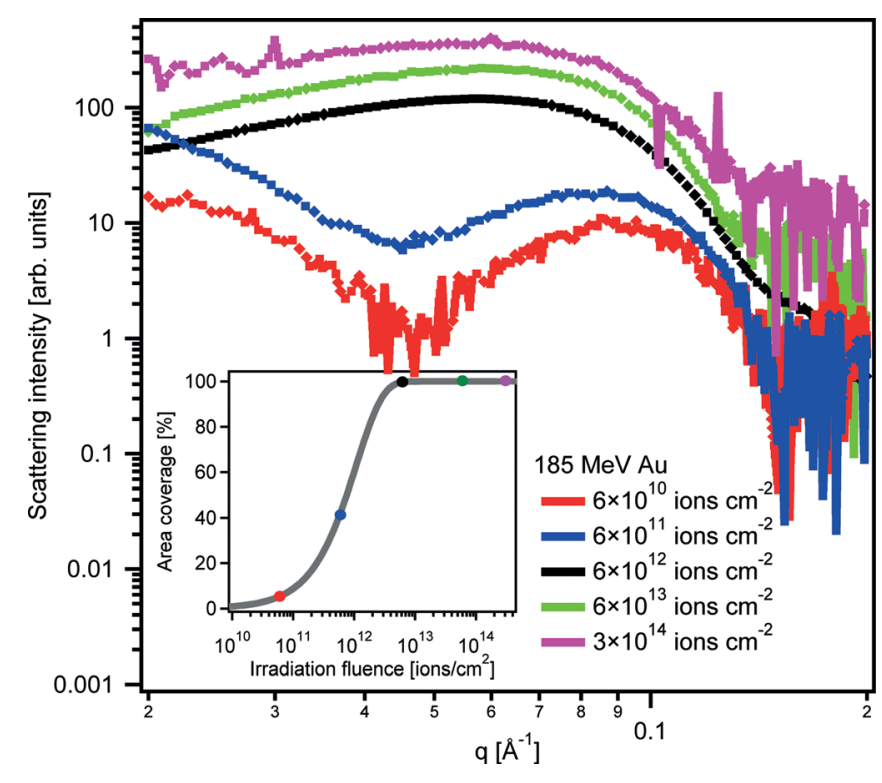

FIG. 1. (Color online) SAXS spectra of a-SiO 2 irradiated with $185 \mathrm{MeV} \mathrm{Au}$ ions as a function of irradiation fluence. The inset shows the areal coverage of the material with ion tracks assuming a track radius of $54 \AA$.

Molecular dynamics simulations were performed using the classical MD code PARCAS. ${ }^{18}$ Sequential simulation of two ion tracks was performed with track center-to-center distances of 8,5 , and $3 \mathrm{~nm}$. Atoms in the cell were shifted between the two simulations, such that both tracks could be simulated at the center of the cell. The initial cell sizes were $17.3 \times 17.3 \times 11.5 \mathrm{~nm}^{3}$ with periodic boundary conditions, cooled with Berendsen temperature control ${ }^{19}$ at the borders toward $0 \mathrm{~K}$. A computation time of $50 \mathrm{ps}$ at $0.4 \mathrm{fs}$ timesteps was used. The atomic interactions were calculated using the Watanabe-Samela Si-O mixed system many-body potential. $^{20,21}$ The electronic energy loss of the SHIs was implemented by an instantaneous deposition of kinetic energy in a random direction for all of the atoms in the simulation cell. The radially symmetrical energy deposition profile with respect to the track center was based on calculations using an inelastic thermal spike model $^{22}$ for a $164 \mathrm{MeV} \mathrm{Au}$ beam in a-SiO ${ }_{2}$. The energy is corresponding to the average ion energy of initially $185 \mathrm{MeV}$ Au ions over the $2 \mu \mathrm{m}$ thick a$\mathrm{SiO}_{2}$ layer. The deposition profile shows a FWHM of approximately $4 \mathrm{~nm}$. We emphasize that kinetic energy is deposited to all of the atoms in the computation cell, however, it is only large enough to cause substantial damage within the first few nanometers around the SHI path. Generation of the $\mathrm{a}-\mathrm{SiO}_{2}$ cells and implementation of the electronic energy loss are described in more detail in Refs. 16 and 23.

\section{RESULTS AND DISCUSSION}

The SAXS intensities of the samples irradiated at $185 \mathrm{MeV}$ at fluences between $6 \times 10^{10}$ and $3 \times 10^{14}$ ions $/ \mathrm{cm}^{2}$ are shown in Fig. 1. A qualitative change and an increase in the scattering intensity are apparent with increasing fluence up to $6 \times 10^{12}$ ions $/ \mathrm{cm}^{2}$. As reported earlier, ${ }^{16}$ at a fluence of $6 \times 10^{10}$ ions $/ \mathrm{cm}^{2}$ the tracks were well separated, i.e., the extent of ion track overlap was negligible and the scattering consistent with a cylindrical core-shell ion
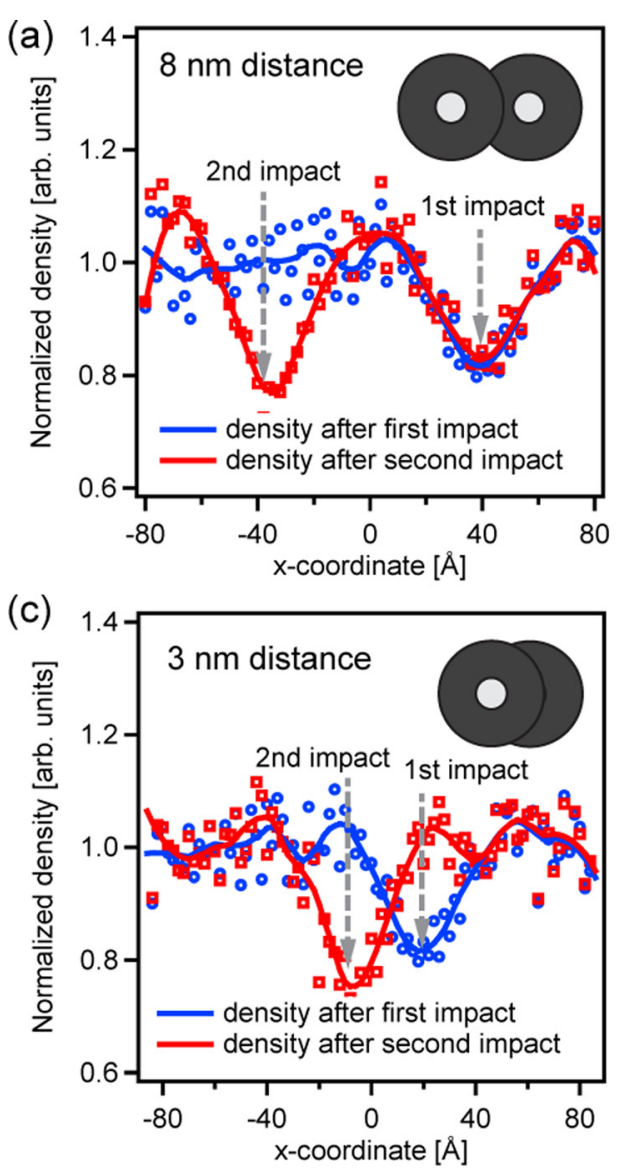

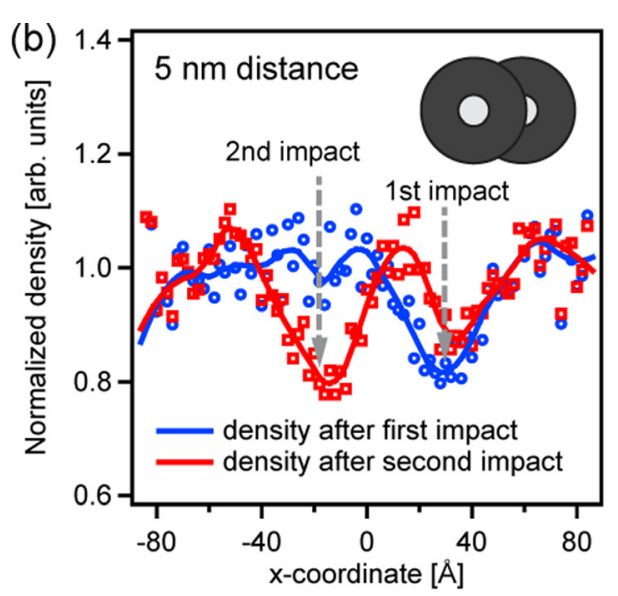

(d)

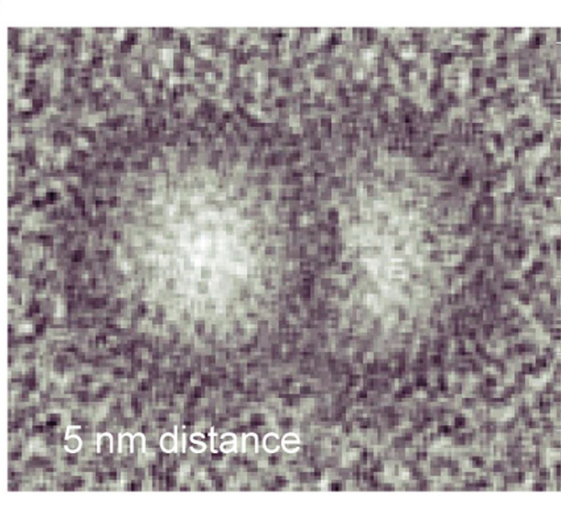

FIG. 2. (Color online) Normalized densities of the $\mathrm{a}-\mathrm{SiO}_{2}$ obtained from MD simulations after subsequent impact of two adjacent ions at distances of (a) 8 $\mathrm{nm}$, (b) $5 \mathrm{~nm}$, and (c) $3 \mathrm{~nm}$. The blue line (circles) shows the densities after the first and the red line (squares) after the second impact. The ion $\mathrm{x}$ coordinates for the first and second ion impact are marked by gray arrows and are (a) $40 \AA$ and $-40 \AA$, (b) $30 \AA$ and $-20 \AA$, and (c) $20 \AA$ and $-10 \AA$, respectively. The top right of the panel shows a schematic cross-section of the two ion tracks drawn to scale using the core and shell dimensions for ion tracks measured using SAXS (Ref. 16). (d) Top view of the simulated densities corresponding to a separation of the tracks of $5 \mathrm{~nm}$. 
track structure with a lower density core and a higher density shell when compared to unirradiated material. Increasing the irradiation fluence leads to increasing coverage of the sample with ion tracks as well as significant track overlap: track overlap is responsible for the qualitative shape change of the scattering spectra, as it ion tracks can no longer be viewed as independent scattering objects. Above a fluence of $6 \times 10^{12}$ ions $/ \mathrm{cm}^{2}$, the scattering becomes nearly identical, i.e., fluence independent. (Note that the intensities for samples irradiated with fluences of $6 \times 10^{13}$ and $3 \times 10^{14}$ ions $/ \mathrm{cm}^{2}$ are offset vertically for better visualization.) The area $A$ covered by ion tracks calculated using a simple overlap model $A=1-\exp \left(-\pi \cdot R^{2} \cdot F\right)$ is shown in the inset of Fig. 1 as a function of ion fluence $F$. The ion track radius $R$ was assumed to be $54 \AA$ at $185 \mathrm{MeV}$ irradiation. ${ }^{16}$ At this energy, a fluence of approximately $5 \times 10^{12}$ ions $/ \mathrm{cm}^{2}$ is required to completely cover the irradiated area with ion tracks, coinciding with the observed value above which the scattering becomes fluence independent. In the following we will concentrate on the fluence range where the material is completely covered with ion tracks. The consequences of the observed scattering in this fluence range are: (i) the material is not homogenously compacted by $\sim 3 \%$ when covered with tracks as inferred from previous studies, ${ }^{4,24}$ in fact it must comprise density fluctuations on the length scale comparable to the ion track dimensions; (ii) these density fluctuations constitute a steady state as the scattering does not change markedly with increasing irradiation fluence once the irradiated area is completely covered with ion tracks. To investigate this behavior, we have performed MD simulations of ion tracks passing in close proximity of an existing ion track. Previous simulations of single ion tracks agreed well with results from SAXS measurements. ${ }^{16,23}$ Sequential simulation of two ion tracks were performed with track center to center distances of 8,5 , and $3 \mathrm{~nm}$. Cross-sections of the resulting density profiles through the center of both tracks, averaged over six simulation runs for all cases to obtain better statistics, are shown in Figs. 2(a)-2(c). The blue lines (circles) show the density after the first simulated ion impact, the red lines (squares) after the second. The x-coordinates of the first and second ion impacts are marked by arrows in Fig. 2 and are $40 \AA$ and $-40 \AA$ in Fig. 2(a), $30 \AA$ and $-20 \AA$ in Fig. 2(b), and $20 \AA$ and $-10 \AA$ in Fig. 2(c), respectively. The top right hand corner of the figure panels show schematics of the top view of two ion tracks where the light and dark areas represent the core and shell of the track, respectively. The schematic is drawn to scale with respect to the distance between the tracks and the core and shell dimensions of the cylindrical ion tracks from previous SAXS measurements for an ion energy of $185 \mathrm{MeV} .{ }^{16}$ We can clearly observe from Fig. 2 (a) that at a distance of $8 \mathrm{~nm}$ the low-density area of the first track core remains unaffected, consistent with no overlap of the high-density shell of the second track. At a distance of $5 \mathrm{~nm}$ [Fig. 2(b)] the density deficit from the first track core is somewhat reduced consistent with the expected partial overlap with the shell from the second ion track. Figure 2(d) shows a top view of the corresponding simulation cell visualizing the overlap of the two impacts, darker colors indicate denser areas. At a distance of $3 \mathrm{~nm}$, schematic and simula- tions agree with an almost complete annihilation of the under-dense core of the first ion track as shown in Fig. 2(c). In all cases is the density distribution of the second ion track radially symmetric, regardless of the preceding local density, as apparent from the example shown in Fig. 2(d). The MD simulations clearly show that low-density areas of an existing track can be filled or "annihilated" by high-density areas of ion tracks that are subsequently generated in close proximity. As apparent from Fig. 2, the distances of the interaction observed in the simulations agree extremely well with the previously measured ion track dimensions (represented in the schematics).

Based on this interaction model, we can now construct a simple picture of the density fluctuations in the material for the given fluences. A simulation of random ion impacts is shown in Fig. 3(a) using the dimensions of the core-shell structure of the ion tracks for the given irradiation conditions, i.e., $16 \AA$ for the core radius and $38 \AA$ for the shell thickness. ${ }^{16}$ The black areas show the unirradiated parts of the material, the gray areas denote the high density regions (corresponding to the shell in an individual ion track), and the white areas the low density regions (corresponding to the core in an individual ion track). Consistent with the MD

(a)

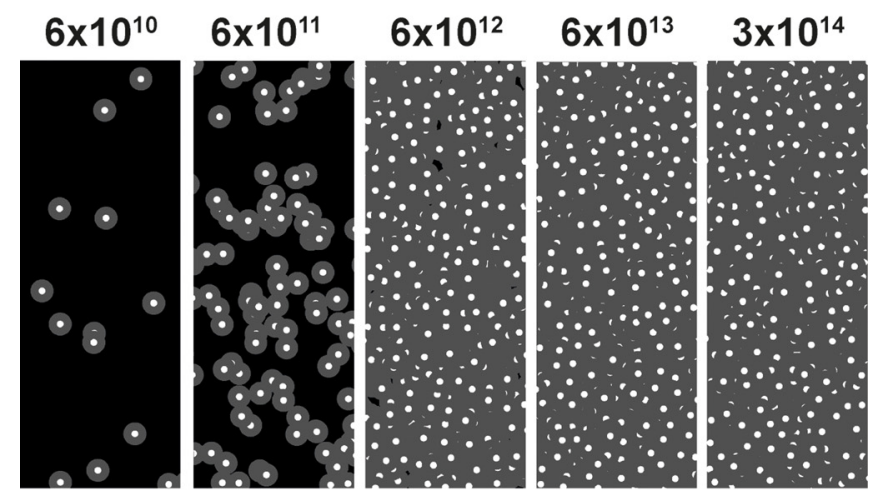

(b)

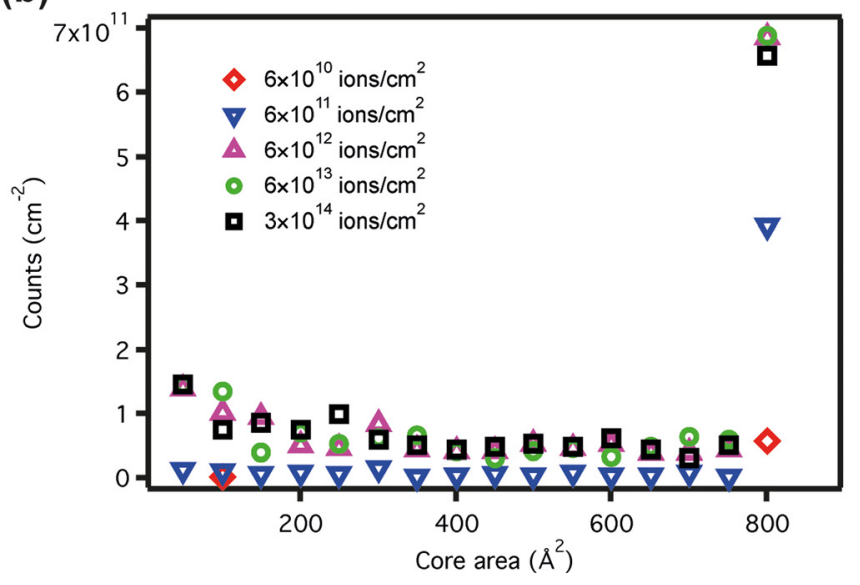

FIG. 3. (Color online) (a) Simulation of random ion impacts for the fluences under investigation. The simulations assume that low-density areas of overlapped tracks are erased. The black areas show the unirradiated parts of the material, the gray areas denote the high density and the white areas the low density regions. The core radius of $16 \AA$ and the shell thickness of $38 \AA$ were taken from Ref. 16. (b) Histograms of the low density areas for the fluences used in (a). 

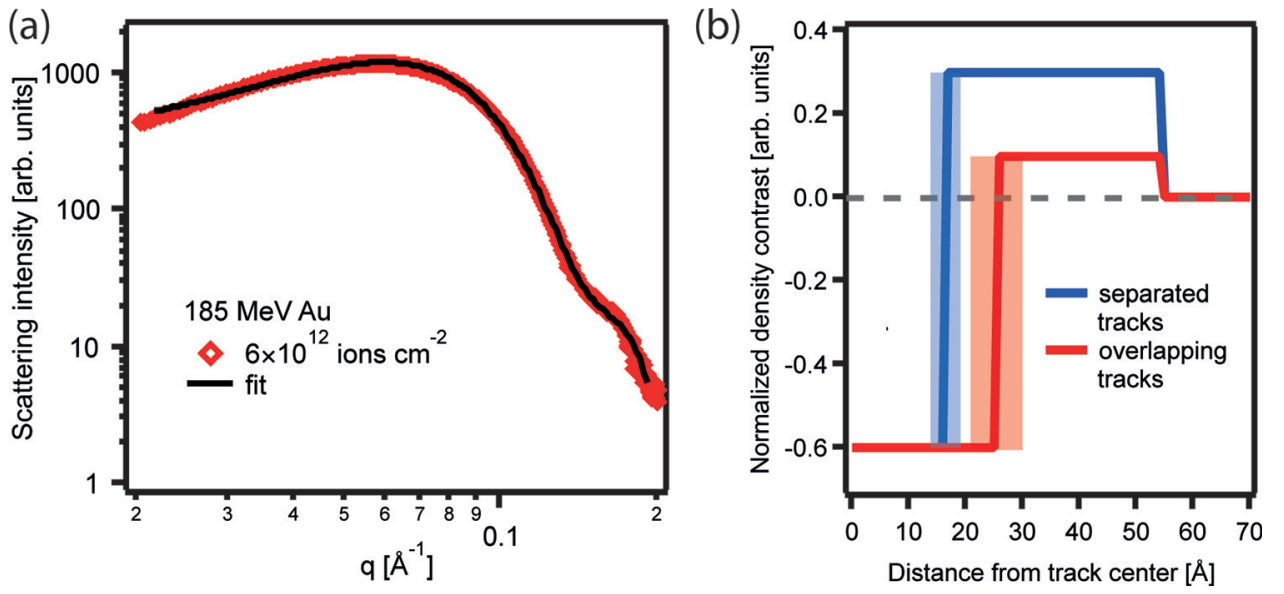

FIG. 4. (Color online) (a) SAXS spectrum and fit for $\mathrm{a}-\mathrm{SiO}_{2}$ irradiated to a fluence of $6 \times 10^{12}$ ions $/ \mathrm{cm}^{2}$. (b) Density profile reconstructed from the fit. For comparison the density profile of a separated track is shown with the dimensions taken from Ref. 16. simulations, new impacts cover existing ones, "annihilating" the density deficit in the low density areas. It is apparent, that at a fluence of $6 \times 10^{12}$ ions $/ \mathrm{cm}^{2}$ the entire area of the sample is essentially covered with ion tracks, in agreement with the overlap calculations shown earlier. Figure 3(b) shows histograms of the low-density areas for all fluences. Two main features can be observed: (i) at $6 \times 10^{12}$ ions $/ \mathrm{cm}^{2}$ and above, the histograms are virtually the same, confirming this model indeed runs into a steady state; (ii) a large number of low density areas approximately retain the original size of an individual track core. Quantitative analysis of the scattering intensities from the high fluence samples is challenging given the complex structure that evolves when tracks overlap. However, with the large number of low density areas retaining the area of individual track cores, a fit with the core-shell model $^{16}$ appears a reasonable approximation to verify if the simulated overlap is consistent with the experimental observation. The model assumes a cylindrically symmetric density distribution, consistent with continuous track formation and approximately uniform energy loss over the depth of the $\mathrm{SiO}_{2}$ layer. A Gaussian distribution of track core radii was assumed to account for deviations from perfectly cylindrical tracks and the distribution of low density areas. The scattering spectrum and the corresponding fit to the core-shell model are shown in Fig. 4(a). The extracted core radius $R_{c}$ and shell thickness $T_{s}$ amount to $26 \pm 1 \AA$ and $28 \pm 1 \AA$. The ratio $\rho_{s}$ between core-matrix and shell-matrix density difference is $-0.18 \pm 0.01$ and the width of the distribution in core radii equals $\sigma=4.5 \pm 0.1 \AA$. Figure 4 (b) plots the corresponding density profile together with that from separated tracks using the values from Ref. 16. The transparent squares show the widths of the distributions in $R_{c}$. The total track radii $R=R_{c}$ $+T_{s}$ of both samples are in excellent agreement, while the core radius of the overlapping track comes out somewhat larger than that of separated tracks. The density ratio $\rho_{s}$ changes from -0.45 to -0.18 from separated to overlapping tracks. While the former is consistent with densification, the latter approximately satisfies the condition for volume conservation. This behavior is in good qualitative agreement with experimental observations of initial compaction of the $\mathrm{SiO}_{2}$ by about $3 \%$ under $\mathrm{SHI}$ irradiation after which the overall density remains constant. MD simulations of multiple tracks also reproduce this compaction, although saturating at about 5\% change. The difference in the track core radii extracted from SAXS between separated and overlapping tracks may be related to a more complicated sample structure after complete track coverage than the model assumes, however, qualitatively, the results are consistent with the proposed and simulated track overlap model.

We note that the observed density fluctuations are not an obvious consequence of the structure of individual ion tracks. The random ion impacts with multiple track overlap could conceivably even out the density difference in individual tracks driven by plastic flow of the a-SiO 2 in the high temperature spikes generated by the SHIs. Another (not observed) scenario could see the material developing porosity such as observed for SHI irradiated amorphous Ge. ${ }^{25}$

\section{CONCLUSIONS}

In conclusion, using small angle x-ray scattering measurements and molecular dynamics simulations, we have demonstrated that swift heavy ion irradiated $\mathrm{a}-\mathrm{SiO}_{2}$ is characterized by density fluctuations on nanometer length scales that resemble a steady state when the material is completely covered with ion tracks. The state is consistent with the assumption that low-density core areas that characterize separated ion tracks are "annihilated" by tracks generated in close proximity. This microscopic view of a steady state of density fluctuations sheds new light on the densification process and the widely accepted macroscopic notion of a homogeneously compacted layer. It is of significance for processes such as the metal nanoparticle deformation and certainly important for optical applications of SHI irradiated a-SiO $\mathrm{S}_{2}$.

\section{ACKNOWLEDGMENTS}

The authors acknowledge the Australian Research Council and the Australian Synchrotron Research Program for financial support and thank the staff at the ANU Heavy Ion facility for their continued technical assistance. O.P., F.D., and K.N. acknowledge financial support from the Academy of Finland under its Centre of Excellence program as well as the OPNA project, and grants of computer capacity from CSC. Part of this research was undertaken at ChemMatCARS Sector 15 at the Advanced Photon Source, USA. ChemMatCARS is principally supported by the 
National Science Foundation/Department of Energy under Grant No. NSF/CHE-0822838. Use of the Advanced Photon Source was supported by the U. S. Department of Energy, Office of Science, Office of Basic Energy Sciences, under Contract No. DE-AC02-06CH11357. Part of this research was undertaken on the SAXS/WAXS beamline at the Australian Synchrotron, Victoria, Australia.

${ }^{1}$ S. Klaumünzer, Mat. Fys. Medd. K. Dan. Vidensk. Selsk. 52, 293 (2006).

${ }^{2}$ M. Lang, F. Zhang, J. Zhang, J. Wang, B. Schuster, C. Trautmann, R. Neumann, U. Becker, and R. C. Ewing, Nat. Mater. 8, 793 (2009).

${ }^{3}$ M.-D. Hou, S. Klaumünzer, and G. Schumacher, Phys. Rev. B 41, 1144 (1990).

${ }^{4}$ S. Klaumunzer, Nucl. Instrum. Methods Phys. Res. B 225, 136 (2004).

${ }^{5}$ W. Wesch, A. Kamarou, and E. Wendler, Nucl. Instrum. Methods Phys. Res. B 225, 111 (2004).

${ }^{6}$ A. Meftah, F. Brisard, J. M. Costantini, E. Dooryhee, M. Hage-Ali, M. Hervieu, J. P. Stoquert, F. Studer, and M. Toulemonde, Phys. Rev. B 49, 12457 (1994).

${ }^{7}$ B. Afra, M. Lang, M. D. Rodriguez, J. Zhang, R. Giulian, N. Kirby, R. C. Ewing, C. Trautmann, M. Toulemonde, and P. Kluth, Phys. Rev. B 83, 064116 (2011).

${ }^{8}$ C. D’Orleans, J. P. Stoquert, C. Estournes, C. Cerruti, J. J. Grob, J. L. Guille, F. Haas, D. Muller, and M. Richard-Plouet, Phys. Rev. B 67, 220101 (2003).

${ }^{9}$ R. Giulian, P. Kluth, L. L. Araujo, D. J. Sprouster, A. P. Byrne, D. J. Cooksen, and M. C. Ridgway, Phys. Rev. B 78, 125413 (2008).

${ }^{10}$ P. Kluth, R. Giulian, D. J. Sprouster, C. S. Schnohr, A. P. Byrne, D. J. Cookson, and M. C. Ridgway, Appl. Phys. Lett. 94, 113107 (2009).

${ }^{11}$ M. C. Ridgway, R. Giulian, D. J. Sprouster, P. Kluth, L. L. Araujo, D. J. Llewellyn, A. P. Byrne, F. Kremer, P. F. P. Fichtner, G. Rizza, H. Amekura, and M. Toulemonde, Phys. Rev. Lett. 106, 095505 (2011).
${ }^{12}$ R. Giulian, P. Kluth, D. J. Sprouster, L. L. Araujo, A. P. Byrne, and M. C. Ridgway, Nucl. Instrum. Methods Phys. Res. B 266, 3158 (2008).

${ }^{13}$ S. Roorda, T. van Dillen, A. Polman, C. Graf, A. van Blaaderen, and B. J. Kooi, Adv. Mater. 16, 235 (2004).

${ }^{14}$ E. A. Dawi, G. Rizza, M. P. Mink, A. M. Vrendenberg, and F. H. P. M. Habraken, J. Appl. Phys. 105, 074305 (2009).

${ }^{15}$ T. van Dillen, E. van der Giessen, P. R. Onck, and A. Polman, Phys. Rev. B 74, 132103 (2006).

${ }^{16}$ P. Kluth, C. S. Schnohr, O. H. Pakarinen, F. Djurabekova, D. J. Sprouster, R. Giulian, M. C. Ridgway, A. P. Byrne, C. Trautmann, D. J. Cookson, K. Nordlund, and M. Toulemonde, Phys. Rev. Lett. 101, 175503 (2008).

${ }^{17}$ P. Kluth, C. S. Schnohr, D. J. Sprouster, A. P. Byrne, D. J. Cookson, and M. C. Ridgway, Nucl. Instrum. Methods Phys. Res. B 266, 2994 (2008).

${ }^{18}$ The main principles of the molecular dynamics algorithms are presented in K. Nordlund et al., Phys. Rev. B 57, 7556 (1998); M. Ghaly et al., Philos. Mag. A 79, 795 (1999); the adaptive time step and electronic stopping algorithms are the same as in K. Nordlund, Comput. Mater. Sci. 3, 448 (1995).

${ }^{19}$ H. J. C. Berendsen, J. P. M. Postma, W. F. van Gunsteren, A. DiNola, and J. R. Haak, J. Chem. Phys. 81, 3684 (1984).

${ }^{20}$ T. Watanabe, D. Yamasaki, K. Tatsumura, and I. Ohdomari, Appl. Surf. Sci. 234207 (2004)

${ }^{21}$ J. Samela, K. Nordlund, V. N. Popok, and E. E. B. Campbell, Phys. Rev. B 77, 075309 (2008).

${ }^{22}$ M. Toulemonde, W. Assman, C. Trautmann, F. Gruner, H. D. Mieskes, H. Kucal, and Z. G. Wang, Nucl. Instrum. Methods Phys. Res. B 212, 346 (2003).

${ }^{23}$ O. H. Pakarinen, F. Djurabekova, K. Nordlund, P. Kluth, and M. C. Ridgway, Nucl. Instrum. Methods Phys. Res. B 267, 1456 (2009).

${ }^{24}$ R. A. B. Devine, J. Non-Cryst. Solids 152, 50 (1993).

${ }^{25}$ T. Steinbach, C. S. Schnohr, P. Kluth, R. Giulian, L. L. Araujo, D. J. Sprouster, M. C. Ridgway, and W. Wesch, Phys. Rev. B 83, 054113 (2011). 